Electrical, Electronics and communications, and Computer Engineering

\title{
Design of a PID-Lead Compensator for a Twin Rotor Aerodynamic System (TRAS)
}

\author{
Rafal Fawzi Faisal * \\ M.Sc. student \\ University of Baghdad/ College of Engineering/ \\ Computer Engineering Department \\ rafal.ff12@gmail.com
}

Omar Waleed Abdulwahhab

Asst. Prof. Dr.

University of Baghdad/ College of Engineering/

Computer Engineering Department

omar.waleed@coeng.uobaghdad.edu.iq

\begin{abstract}
This paper deals with a Twin Rotor Aerodynamic System (TRAS). It is a Multi-Input MultiOutput (MIMO) system with high crosscoupling between its two channels. It proposes a hybrid design procedure that combines frequency response and root locus approaches. The proposed controller is designated as PID-Lead Compensator (PIDLC); the PID controller was designed in previous work using frequency response design specifications, while the lead compensator is proposed in this paper and is designed using the root locus method. A general explicit formula for angle computations in any of the four quadrants is also given. The lead compensator is designed by shifting the dominant closed-loop poles slightly to the left in the s-plane. This has the effect of enhancing the relative stability of the closed-loop system by eliminating the oscillation in its transient part but at the expense of greater rise time. However, for some applications, long rise time may be an allowable price to get rid of undesired oscillation. To demonstrate the proposed hybrid controller's performance numerically, a new performance index, designated by Integral Reciprocal Time Absolute Error (IRTAE), is defined as a figure to measure the oscillation of the response in its transient part. The proposed controller enhances this performance index by $0.6771 \%$. Although the relative enhancement of the performance index is small, it contributes to eliminating the oscillation of the response in its transient part. Simulation results are performed on the MATLAB/Simulink environment. Keywords: TRAS, Twin Rotor Aerodynamic System, PID controller, frequency response specifications, lead compensator.
\end{abstract}

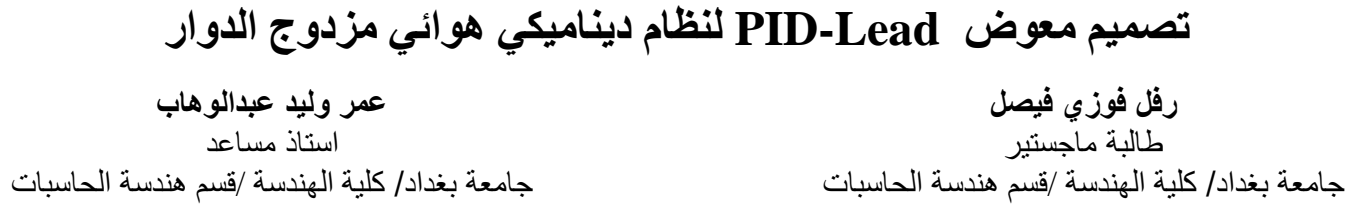

الخلاصة

يتعامل هذا البحث مع النظام الديناميكي الهوائي مزدج الدوار (TRAS)، وهو نظام ذات ادخات ادخالات متعددة واخراجات

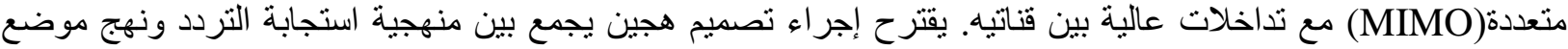
الجذر. المسبطر المقترح صمح كمعوض PIDLC) PID-Lead) ؛ تم تصميم المسيطر التناسبي و التكاملي والتفاضلي في فئي

*Corresponding author

Peer review under the responsibility of University of Baghdad.

https://doi.org/10.31026/j.eng.2021.01.06

2520-3339 (C) 2019 University of Baghdad. Production and hosting by Journal of Engineering.

This is an open access article under the CC BY4 license http://creativecommons.org/licenses/by /4.0/).

Article received: $14 / 6 / 2020$

Article accepted: 30/7/2020

Article published:1/1/2021 


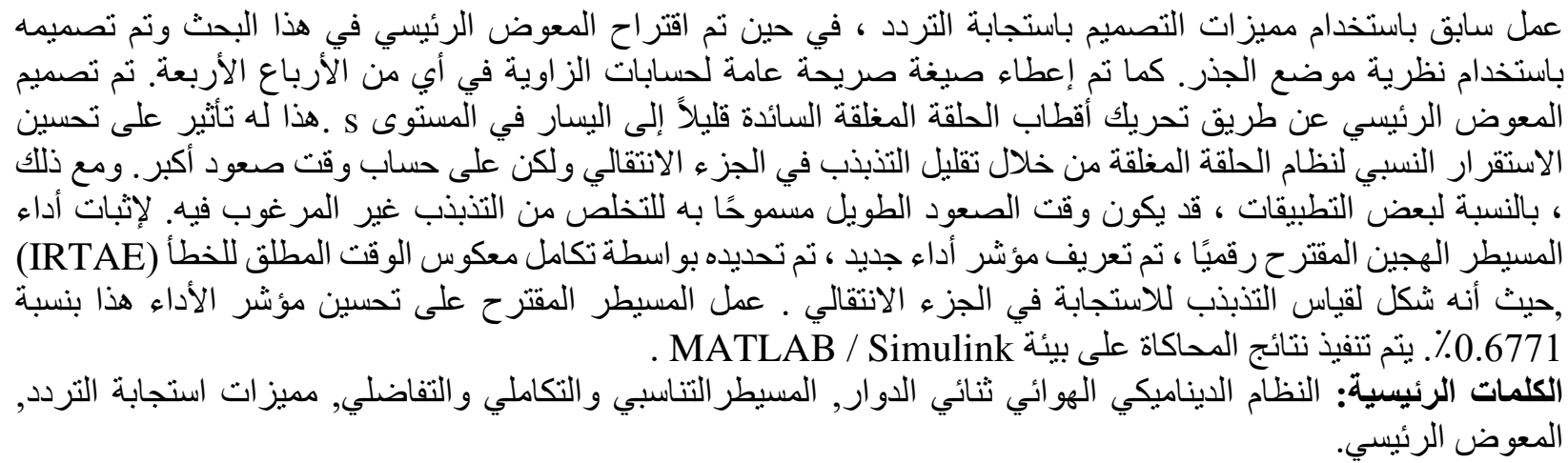

\section{INTRODUCTION}

The Twin Rotor Aerodynamic System (TRAS) is a lab machine that behaves like a helicopter; thus, it is an important issue to design a system controller. It consists of two propellers tied to the ends of a beam. These propellers are driven by two DC motors Fig. 1. The main rotor generates vertical pushes and rotation around the horizontal axis, and the tail rotor generates horizontal pushes and rotation around the vertical axis (Netto, Lakhani and Meenatchi Sundaram, 2019). The TRAS is a nonlinear system with high nonlinearity and high coupling interaction between its two channels. It is a Multi-Input Multi-Output (MIMO) system where it has two inputs $u_{\phi}$, $u_{\theta}$ The voltages to the DC motors are connected to the tail, and main rotors, respectively, and have two outputs $\phi$ and $\theta$, which are the angles from the tail and main rotors, respectively (Haruna $\boldsymbol{e t}$ al., 2019).

Designing a controller for a nonlinear MIMO system with high coupling is a complex task. Therefore some simplification has to be done, such as linearizing the system. Many attempts have been made to control the TRAS. In (Abdulwahhab and Abbas, 2017) and referred in (Abdulwahhab and Abbas, 2020), a fractional-order Proportional-Integral-Derivative (FOPID) controller $\left(\mathrm{PI}^{\lambda} \mathrm{D}^{\mu}\right)$ of TRAS has been proposed with a new method called a Resonant Peak Value Design for the magnitude curve of the open-loop transfer function, where the FOPID controller performed better than the PID controllers in performance and robustness. In (Coskunturk, 2018), two controllers have been used, the Sliding Mode Controller (SMC) and Linear Quadratic Regulator (LQR). The mathematical model of the TRAS system has been created based on Lagrange's Equations. The simulated results have shown that both controllers worked synchronously, but monitoring efficiency can be improved by changing the parameters. Many developments may be proposed to increase the stability of the system. The system should include the Kalman Filter. The Linear Quadratic Regulator is converted to Linear Quadratic Gaussian because it reduces the sliding mode control track's oscillations. In (Chaudhary and Kumar, 2019), two Degrees Of Freedom (DOF) PID controller and FOPID have been designed for TRAS. The controllers were designed based on tuning methods. The final result showed that FOPID is better than the 1-DOF PID controller and 2-DOF PID controller to improve TRAS's steady-state response and transient response. In (Rojas-Cubides et al., 2019), an SMC scheme assisted by Generalized Proportional Integral Observers (GPI) was proposed for TRAS. The proposed technique has shown to sustain the sliding system even in unfavorable operating environments, for example, parameter fluctuations, external disturbances, nonlinear effects, and actuator failures. This illustrates the robustness and practical efficiency of the proposed strategy. The most important features of the strategy used are (a) For each decoupling loop, only the order and function $K_{i}(t, y)$ are required, (b) A single GPI observer for each uncoupling loop can be used to estimate the conditions and disturbances associated with each sliding surface, (c) The architecture of each control law must be reduced to a single coefficient deciding the dynamics of the tracking error within the sliding surface. In (Castillo, Kutlu, and Atan, 2020), two intuitionistic fuzzy 
controllers were designed separately, one for the main rotors and the other is for the tail rotors. The output was obtained from combining them. Because of the MIMO twin-rotor system configuration, it has many uncertainties. The intuitionistic fuzzy control provides an alternative to the optimal PID method under system uncertainties. The Simulation results showed that intuitionistic fuzzy control increases the reference signal's efficiency according to the PID system. The control method is designed to provide good performance on highly nonlinear systems, in which uncertainties are often modeled. In (Hassan, Hossam and El-Badawy, 2020), robust $\mathrm{H}_{\infty}$ controller was designed. The nonlinear mathematical model of TRAS was obtained by using Euler's law and then linearized about an equilibrium point. The proposed control strategies for trajectory tracking can be shown to be successful. In (Goyal $\boldsymbol{e t}$ al., 2020), a robust Linear Matrix Inequality (LMI) based PI controller was designed for tracking control of a TRAS. The system model was represented in a linear form from nonlinear that does not involve dropping any higherorder term. The proposed controller with a decentralized structure was contributed to removing the cross-coupling between two TRAS channels. Furthermore, it was provided a better setpoint tracking result in good robust performance.

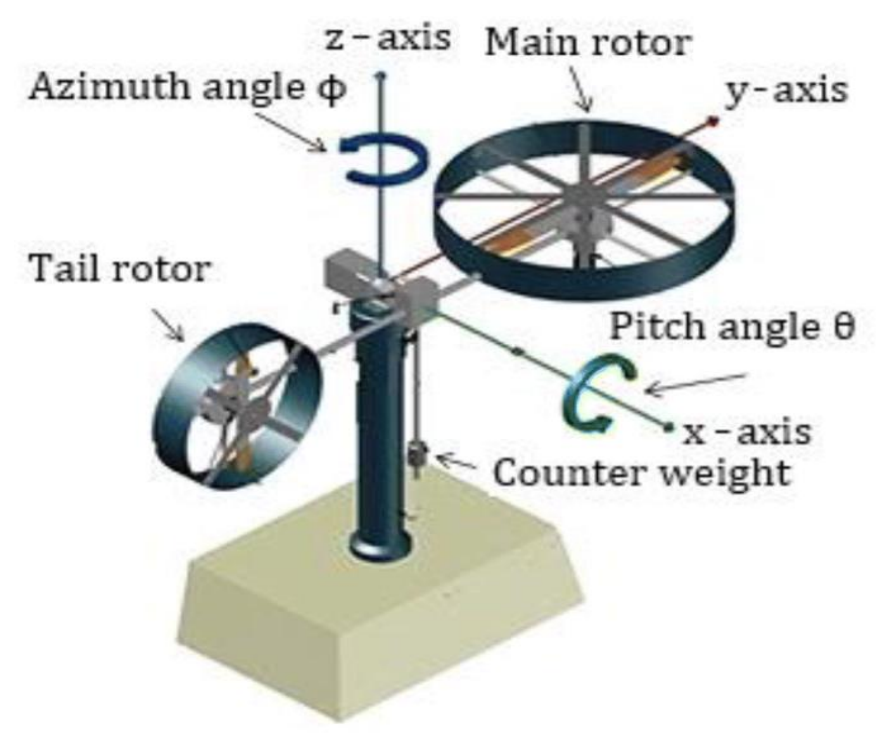

Figure 1. Block diagram of the TRAS system.

The rest of this paper is organized as follows. Section 2 describes the TRAS's mathematical model; Section 3 presents an overview of the PID controller design and the design of a Lead compensator; experimental results are presented and discussed in Section 4, and concluding remarks are given in Section 5.

\section{TRAS MODEL}

A Lagrange's equations are used to derive the equations that represent the dynamics of the TRAS. The mathematical model of the TRAS is given by:

$$
\begin{aligned}
& J_{\phi} \ddot{\phi}=F_{\mathrm{t}}\left(\omega_{t}\right) l_{\mathrm{t}} \cos \theta-\left(c_{\phi} \dot{\phi}+k_{\phi} \phi\right)+J \dot{\phi} \dot{\theta} \sin (2 \theta) \\
& J_{\theta} \ddot{\theta}=F_{\mathrm{m}}\left(\omega_{\mathrm{m}}\right) l_{\mathrm{m}}-\left(c_{\theta} \dot{\theta}+k_{\theta} \theta\right)-\left(k_{1} \cos \theta+k_{2} \sin \theta\right)-J \frac{\dot{\phi}^{2}}{2} \sin (2 \theta)
\end{aligned}
$$


The parameters $J_{\phi}$ and $J_{\theta}$ represent the sums of moments of inertia relative to the vertical axis and the horizontal axis, respectively; they are:

$J_{\phi}=m_{\mathrm{m}} l_{\mathrm{m}}^{2} \cos ^{2} \theta+m_{\mathrm{t}} l_{\mathrm{t}}^{2} \cos ^{2} \theta+2 m_{\mathrm{cw}} l_{\mathrm{cw}}^{2} \sin ^{2} \theta=J \cos ^{2} \theta+J_{A}$

$J_{A}=m_{\mathrm{cw}} l_{\mathrm{cw}}^{2}$

$J=m_{\mathrm{m}} l_{\mathrm{m}}^{2}+m_{\mathrm{t}} l_{\mathrm{t}}^{2}-m_{\mathrm{cw}} l_{\mathrm{cw}}^{2}$

$J_{\theta}=m_{\mathrm{m}} l_{\mathrm{m}}^{2}+m_{\mathrm{t}} l_{\mathrm{t}}^{2}+m_{\mathrm{cw}} l_{\mathrm{cw}}^{2}$

$k_{1}=g\left(\left(m_{\mathrm{m}} l_{\mathrm{m}}-m_{\mathrm{t}} l_{\mathrm{t}}\right)\right.$

$k_{2}=g m_{\mathrm{cw}} l_{\mathrm{cw}}$

where:

$m_{\mathrm{m}}=$ the mass of the main rotor

$m_{\mathrm{t}}=$ the mass of the tail rotor

$m_{\mathrm{cw}}=$ the mass of the point mass mounted at the end of the counterweight rods.

$F_{\mathrm{t}}=$ propulsive force for the tail rotor

$F_{\mathrm{m}}=$ propulsive force for the main rotor

$\omega_{t}=$ the angular velocity of the tail rotor

$\omega_{\mathrm{m}}=$ the angular velocity of the main rotor

$c_{\phi}=$ coefficient of velocity-proportional friction torque in the horizontal plane

$k_{\phi}=$ coefficient of restoring torque in the horizontal plane

$c_{\theta}=$ coefficient of velocity-proportional friction torque in the vertical plane

$k_{\theta}=$ coefficient of restoring torque in the vertical plane

The differential equations Eq.(1) and Eq.(2) are nonlinear in $\phi$ and $\theta$ and have cross-coupling terms. The differential equations describe the dynamics of the tail and main motors:

$\dot{\omega}_{\mathrm{t}}=-\frac{1}{T_{\mathrm{t}}} \omega_{\mathrm{t}}+\frac{g_{\mathrm{t}}}{T_{\mathrm{t}}} u_{\mathrm{t}}$

$\omega_{\mathrm{m}}=-\frac{1}{T_{\mathrm{m}}} \omega_{m}+\frac{g_{\mathrm{m}}}{T_{\mathrm{m}}} u_{\mathrm{m}}$

where :

$T_{\mathrm{t}}$ is the time constant of the tail rotor

$g_{\mathrm{t}}$ is the DC gain of the tail rotor

$u_{\mathrm{t}}$ is the input voltage applied to the tail rotor

$T_{\mathrm{m}}$ is the time constant of the main rotor

$g_{\mathrm{m}}$ is the DC gain of the main rotor

$u_{\mathrm{m}}$ is the input voltage applied to the main roto

Defining a state vector, an input vector, and an output vector respectively as:

$\boldsymbol{x}=\left[\phi, \dot{\phi}, \omega_{\mathrm{t}}, \theta, \dot{\theta}, \omega_{\mathrm{m}}\right]^{T}$

$\boldsymbol{u}=\left[u_{\phi} u_{\theta}\right]^{T}$

$\boldsymbol{y}=[\phi \theta]^{T}$ 
The state space equation is $\dot{\boldsymbol{x}}=\boldsymbol{f}(\boldsymbol{x}, \boldsymbol{u})$ and it is :

$$
\dot{\boldsymbol{x}}=\left[\begin{array}{c}
x_{2} \\
\frac{1}{J \cos ^{2} x_{4}+J_{A}}\left(F_{\phi}\left(x_{3}\right) l_{t} \cos x_{4}-c_{\phi} x_{2}-k_{\phi} x_{1}+J x_{2} x_{5} \sin 2 x_{4}\right) \\
-\frac{1}{T_{1}} x_{3}+\frac{1}{c_{1} T_{1}} u_{\phi} \\
x_{5} \\
\frac{1}{J_{\theta}}\left(F_{\theta}\left(x_{6}\right) l_{m}-k_{1} \cos x_{4}-k_{2} \sin x_{4}-\frac{J}{2} x_{2}^{2} \sin 2 x_{4}-c_{\theta} x_{5}-k_{\theta} x_{4}\right) \\
-\frac{1}{T_{2}} x_{6}+\frac{1}{c_{2} T_{2}} u_{\theta}
\end{array}\right]
$$

and the output equation is:

$\boldsymbol{y}=\left[\begin{array}{llllll}1 & 0 & 0 & 0 & 0 & 0 \\ 0 & 0 & 0 & 1 & 0 & 0\end{array}\right]\left[\begin{array}{llll}x_{1} x_{2} & x_{3} x_{4} x_{5} x_{6}\end{array}\right]^{T}$

\section{DESIGN OF A LEAD COMPENSATOR}

The proposed hybrid controller, the PIDLC, has two parts. The first part is a PID controller, and the second part is a Lead compensator that is proposed in this paper using the root locus method. The PID controller was designed in (Abdulwahhab and Abbas, 2017) and (Abdulwahhab, 2018) using frequency response specifications for a linearized decoupled TRAS model; its nonlinear differential equation was linearized about certain operating point, and the controller was tuned based on the linearized mode.

Linearizing the system at the operating $\operatorname{point}\left(\phi, \dot{\phi}, \omega_{\mathrm{t}}, \theta, \dot{\theta}, \omega_{\mathrm{m}}, u_{\mathrm{t}}, u_{\mathrm{m}}\right)=$ $(0,0,0,-0.7098,0,0,0,0)$ results in a linear decoupled MIMO system. The transfer function matrix of the linearized decoupled TRAS is :

$G(s)=\left[\begin{array}{cc}\frac{9.2461}{(s+0.2)\left(s^{2}+1.27 s+7.619\right)} & 0 \\ 0 & \frac{3.7265}{(s+0.4)\left(s^{2}+0.179 s+3.126\right)}\end{array}\right]$

The PID controller equation is: $u(t)=K_{P}(e)+K_{I} \int_{0}^{t}(e)+K_{D} \frac{d}{d x}(e)$ where $K_{P}, K_{I}, K_{D}>0$.

The control system was designed as a two SISO system, as shown in Fig. 3.

where $G_{11}(s)$ and $G_{22}(s)$ are the diagonal elements of the transfer function matrix in Eq. (10). $G_{11}(s)$ represents the decoupled transfer function of the azimuth angle $\phi$ (horizontal subsystem), which is controlled by the controller $\operatorname{PID}_{\phi}(s)$ and $G_{22}(s)$ represents the decoupled transfer function of the pitch angle $\theta$ (vertical subsystem), which is controlled by the controller $\operatorname{PID}_{\theta}(s) ; \operatorname{PID}_{\phi}(s)$ and $\operatorname{PID}_{\theta}$ were designed in (Abdulwahhab and Abbas, 2017) (Abdulwahhab, 2018) and are given by:

$$
\begin{aligned}
& \operatorname{PID}_{\phi}(s)=\frac{0.1032 s^{2}+0.1909 s+0.05095}{0.1899 s^{2}+s} \\
& \operatorname{PID}_{\theta}(s)=\frac{0.02984 s^{2}+0.1 s+0.07334}{0.108 s^{2}+s}
\end{aligned}
$$




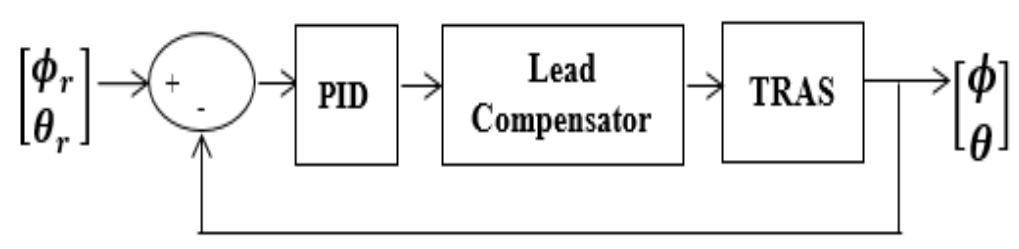

Figure 2. Block Diagram of the TRAS Feedback Control System.

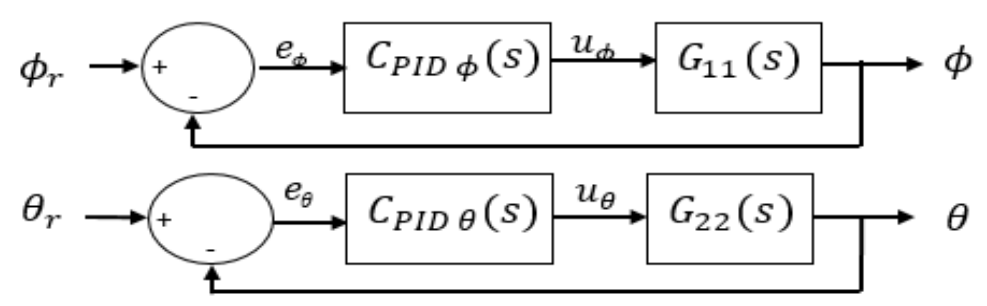

Figure 3. TRAS in Linearized decoupled model.

To design a lead compensator using root locus method, the following procedure was adopted:

Step 1: The values of poles and zeros of the open-loop transfer function are represented in Fig. 4, then a suitable desired point $(\mathrm{Pd})$, which represents a dominant closed-loop pole, is suggested to shift the critical pole to the left. Let $P_{1}=-c+j d, P_{2}=-e+j f, P_{3}=-g+j h$, and $P_{4}=-m+$ jn be open-loop poles or zero.

Step 2: Determining if the angle from a pole or zero to the desired dominant closed-loop pole. It is measured counterclockwise (Ogata, 2009), as shown in Fig. 4. The $\theta_{1}, \theta_{2}, \theta_{3}$ and $\theta_{4}$ are :

$$
\begin{array}{ll}
\theta_{1}=180^{\circ}-\tan ^{-1} \frac{b-d}{a-c} & \text { if } a>c \text { and } b>d \\
\theta_{2}=\tan ^{-1} \frac{b-f}{e-a} & \text { if } e>a \text { and } b>f \\
\theta_{3}=180^{\circ}+\tan ^{-1} \frac{h-b}{a-g} & \text { if } a>g \text { and } b<h \\
\theta_{4}=360^{\circ}-\tan ^{-1} \frac{n-b}{m-a} & \text { if } a<m \text { and } b<n
\end{array}
$$

Step 3: The lead compensator has a transfer function $C(s)=K_{c} \frac{\left(s+Z_{c}\right)}{\left(s+P_{c}\right)}$ where $Z_{c}<P_{c}$. The lead compensator zero must be to the right of the lead compensator pole, as shown in Fig. 5. 


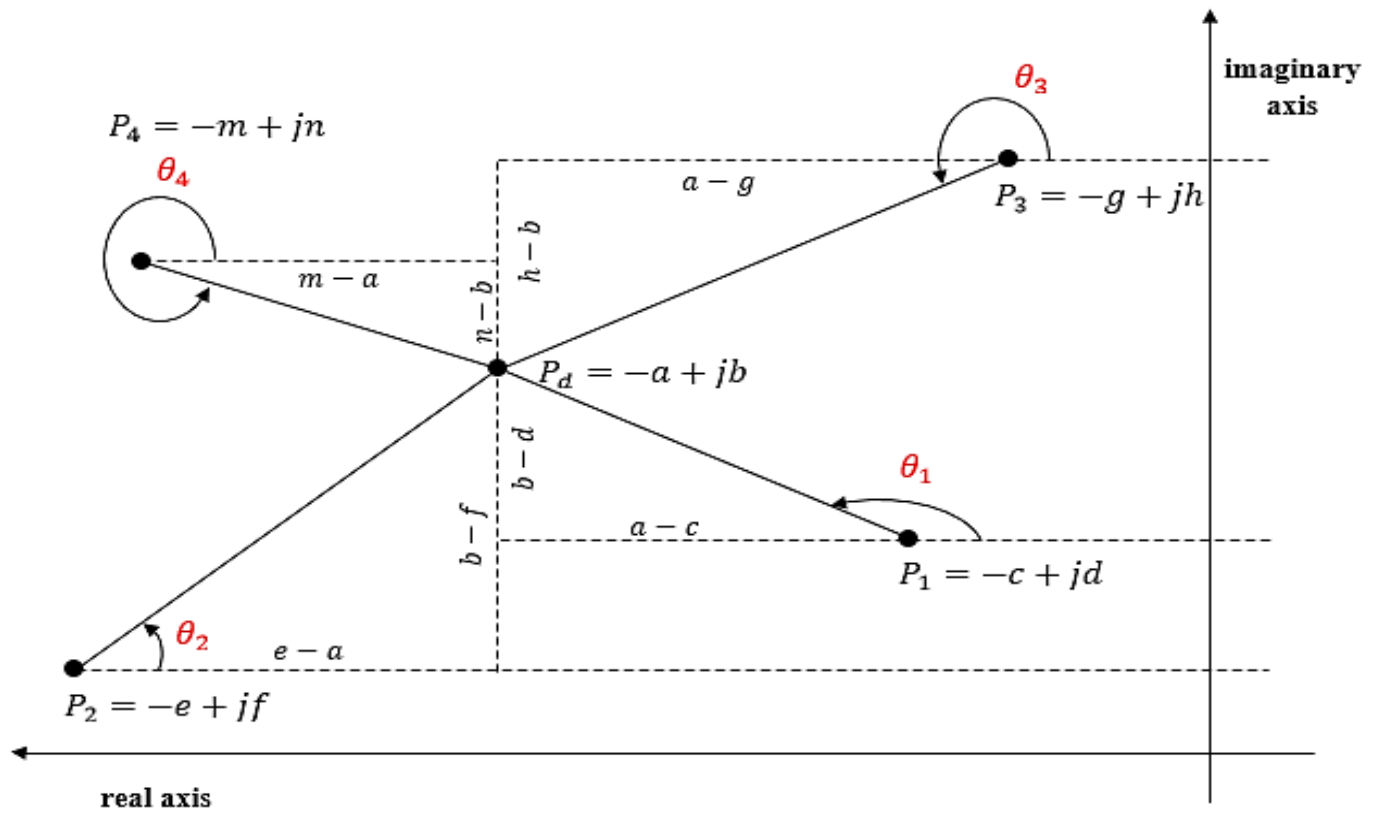

Figure 4. Desired closed-loop pole and open-loop Poles and Zeroes.

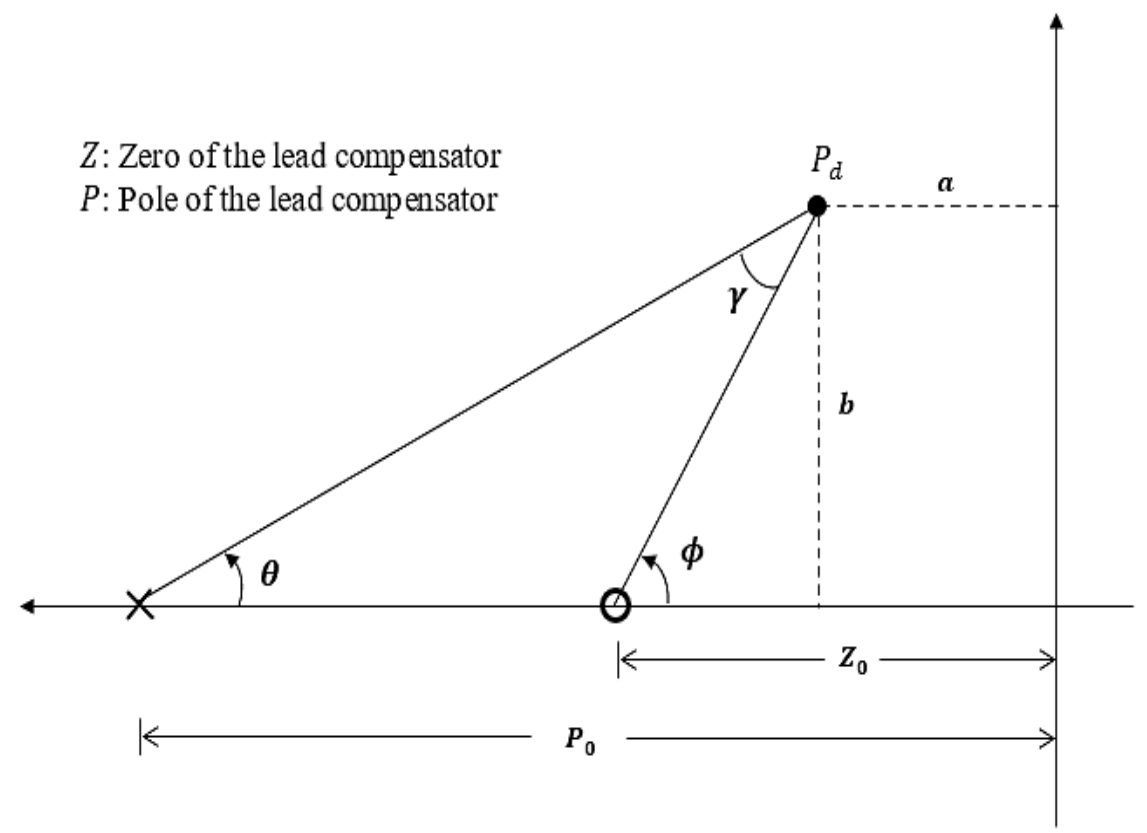

Figure 5. Illustration of $Z_{\mathrm{c}}$ and $P_{\mathrm{c}}$.

Step 4: Calculating the angle of deficiency. The lead compensator must contribute this angle if the new root locus is to pass through the desired locations for the dominant closed-loop poles (Ogata, 2009). Firstly, find the summation of open-loop poles and zeroes angle with respect to the dominant point as:

$$
-\sum \angle \text { poles }+\sum \angle \text { zeros }=\psi
$$

The resultant angle $\psi$ must be negative. Secondly, calculate deficiency angle where: 
Deficiency angle $=180^{\circ}-\psi=-\gamma$

After that, the sum of compensator poles and zeroes must be determined. From the low of the sum of the triangle's angles:

$\theta+\gamma+(180-\phi)=180^{\circ}$

So, $\phi=\theta+\gamma$

Step 5: The location of the zero $s=-Z_{\mathrm{c}}$ can be chosen arbitrarily. The location of the pole $s=$ $-P_{c}$ can be determined as follows:

$\phi=\tan ^{-1} \frac{b}{z_{c}-a}$

$\tan \theta=\tan (\phi-\gamma)=\frac{b}{P_{c}-a}$

$P_{c}=\frac{\mathrm{b}}{\tan (\phi-\gamma)}+a$

Step 6: Finally, determining the value of the lead compensator gain $K_{c}$ from the magnitude condition:

$K_{c}=\frac{1}{\left|G(s) \cdot P l D(s) \cdot \frac{\left(s+Z_{C}\right)}{\left(s+P_{c}\right)}\right|}$

In this paper, $z_{c}$ has been chosen arbitrary equal to 0.1 , since this value is small enough to make the rule of the lead compensator $Z_{c}<P_{c}$. Utilizing equations Eq. (23) and Eq. (24) to design the remaining compensator parameters $P_{c}$ and $K_{c}$, respectively, the resultant compensator parameters for the two channels are shown in Table 1. Thus, the two compensators are:

$C_{\phi}=\frac{0.1438 *(s+0.1)}{(s+0.1321)}$

$C_{\theta}=\frac{0.4603 *(s+0.1)}{(s+0.1272)}$

Using compensator design, the close loop's imaginary poles were shifted to the left to make the TRAS more stable compared with TRAS without the compensator.

\section{SIMULATION RESULTS AND DISCUSSION}

A simulation was carried out using MATLAB/Simulink tool. The closed-loop system's responses with designed PIDLC obtained, as shown in Fig. 6, where the controller was applied to the nonlinear decoupled TRAS system.

From Fig. 6, it is clear that the oscillation of response was eliminated, and the relative stability of the system was increased so that the PIDLC outperforms the PID controller. As a result, the poles in close loop pulls to the left, as shown in Table 1. 
A new performance index, designated by Integral Reciprocal-Time Absolute Error (IRTAE), is proposed in this paper. It is defined by:

$\operatorname{IRTAB}=\int_{0}^{\infty} \frac{1}{t}|e(t)| d t$

where $\epsilon$ is a sufficiently small positive number. In this paper, $\epsilon=0.001$. IRTAE is a measure of how much the system oscillates in its transient part. For the PID based control system, its value is 5.612, while for the PIDLC based system, its value is 5.574; thus, the percentage improvement in this performance index is $0.6771 \%$.

Table 1. The values of TRAS with and without Lead compensator.

\begin{tabular}{|c|c|c|c|c|c|c|c|}
\hline \multirow[b]{2}{*}{ Channel } & \multicolumn{3}{|c|}{ Without compensator } & \multicolumn{4}{|c|}{ With compensator } \\
\hline & Open-loop Poles & $\begin{array}{c}\text { Open-loop } \\
\text { Zeros }\end{array}$ & Closed-loop Poles & Closed-loop Poles & $Z_{c}$ & $P_{c}$ & $K_{c}$ \\
\hline$\phi$ & $\begin{array}{c}0 \\
-5.2659 \\
-0.1764+1.4441 \mathrm{i} \\
-0.1764-1.4441 \mathrm{i} \\
-0.2\end{array}$ & $\begin{array}{l}-1.5264 \\
-0.3235\end{array}$ & $\begin{array}{c}-5.2919 \\
-0.0899+1.4816 \mathrm{i} \\
-0.0899-1.4816 \mathrm{i} \\
-0.1735+0.1214 \mathrm{i} \\
-0.1735-0.1214 \mathrm{i}\end{array}$ & $\begin{array}{c}-5.2661 \\
-0.1542+1.4344 \mathrm{i} \\
-0.1542-1.4344 \mathrm{i} \\
-0.9674 \\
-0.1294+0.0602 \mathrm{i} \\
-0.1294-0.0602 \mathrm{i}\end{array}$ & 0.1 & 0.1321 & 0.1438 \\
\hline$\theta$ & $\begin{array}{c}0 \\
-9.2593 \\
-0.0895+2.1037 \mathrm{i} \\
-0.0895-2.1037 \mathrm{i} \\
-0.3999\end{array}$ & $\begin{array}{l}-2.2671 \\
-1.0841\end{array}$ & $\begin{array}{c}-9.2674 \\
-0.0495+2.1131 \mathrm{i} \\
-0.0495-2.1131 \mathrm{i} \\
-0.2360+0.0738 \mathrm{i} \\
-0.2360-0.0738 \mathrm{i}\end{array}$ & $\begin{array}{c}-9.2630 \\
-0.0712+2.1080 \mathrm{i} \\
-0.0712-2.1080 \mathrm{i} \\
-0.3451 \\
-0.1656 \\
-0.0495\end{array}$ & 0.1 & 0.1272 & 0.4603 \\
\hline
\end{tabular}
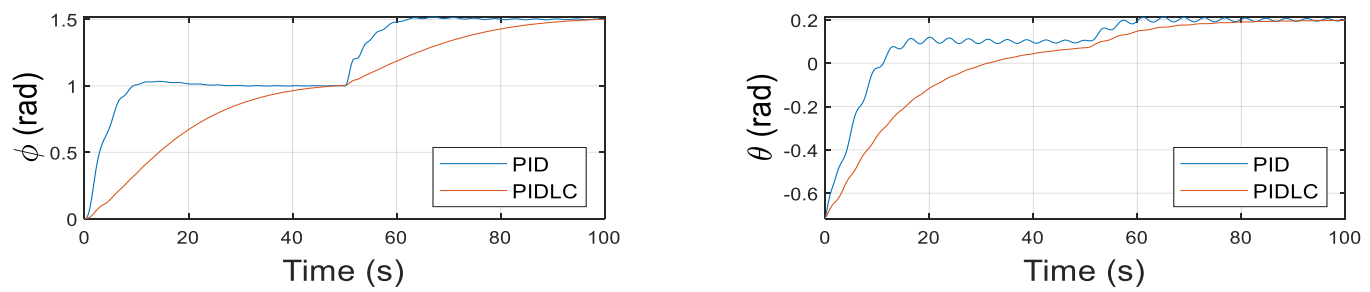

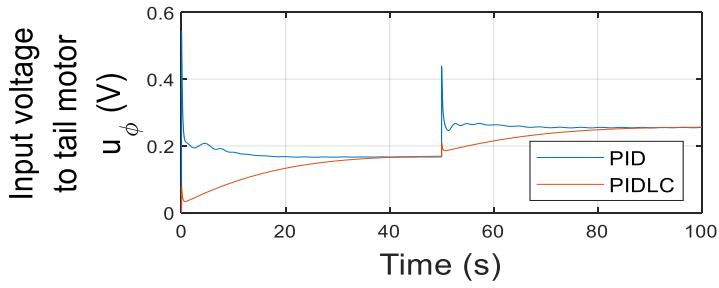

(a)

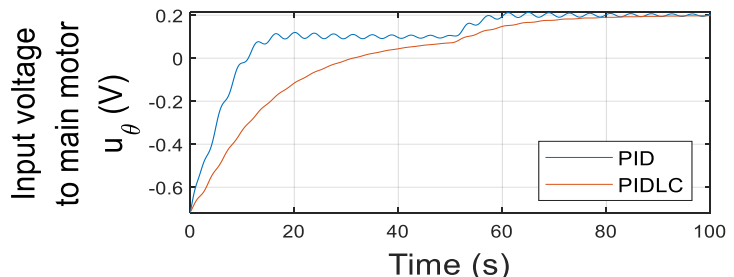

(b)

Figure 6. Step response of the TRAS with PID and PIDLC for (a) Azmith angle and input voltage to the tail rotor (b) Pitch angle and input voltage to the main rotor.

\section{CONCLUSIONS}

In this paper, a hybrid controller is proposed, which is a PID-Lead compensator (PIDLC) by combining frequency response and root locus design methods. The PIDLC enhances the relative stability of the control system compared to the original PID controller. This was demonstrated by enhancing the IRTAE performance index's value, which measures the oscillation of the system's 
transient response. However, the proposed controller increases the rise time, which is considered a drawback. The trade-off between relative stability and rise time can be further investigated in future work.

\section{REFERENCES}

- Abdulwahhab, O. W., 2018. Design and Stability Analysis of Fractional-Order Controllers for MIMO Nonlinear Systems. University of Baghdad.

- Abdulwahhab, O. W., and Abbas, N. H., 2017. A New Method to Tune a Fractional-Order PID Controller for a Twin Rotor Aerodynamic System, Arabian Journal for Science and Engineering. Springer Berlin Heidelberg, 42(12), pp. 5179-5189.

- Abdulwahhab, O. W., and Abbas, N. H., 2020 Survey Study of Factional Order Controllers, Journal of Engineering. Baghdad University, 26(4), pp. 188-201.

- Castillo, O., Kutlu, F., and Atan, Ö., 2020. Intuitionistic fuzzy control of twin rotor multiple input multiple output systems, Journal of Intelligent and Fuzzy Systems. IOS Press, 38(1), pp. 821-833.

- Chaudhary, S., and Kumar, A., 2019. Control of Twin Rotor MIMO System Using 1Degree-of-Freedom PID, 2-Degree-of-Freedom PID and Fractional order PID Controller', 2019 3rd International Conference on Electronics, Communication and Aerospace Technology (ICECA). IEEE, pp. 746-751.

- Coskunturk, Y., 2018. Design of a Robust Controller Using Sliding Mode for Two Rotor Aero-Dynamic System, International Journal of Innovative Technology and Interdisciplinary Sciences, 2(1), pp. 119-132.

- Goyal, J. K. et al.,. 2020 Experimental Design of Robust Decentralized PI Controller for TRMS through Polytopic Modeling, in 2020 IEEE International Conference on Industrial Technology (ICIT). IEEE, pp. 47-52.

- Haruna, A. et al., 2019 Improved integral backstepping control of variable speed motion systems with application to a laboratory helicopter, ISA Transactions. Elsevier Ltd. DOI: 10.1016/j.isatra.2019.07.016.

- Hassan, R., Hossam, A., and El-Badawy, A., 2020. Robust H-infinity Control for a Birotor System, in AIAA Scitech 2020 Forum, p. 1834.

- Netto, W., Lakhani, R., and Meenatchi Sundaram, S., 2019. Design and performance comparison of different adaptive control schemes for pitch angle control in a Twin-RotorMIMO-System, International Journal of Electrical and Computer Engineering, 9(5), pp. 4114-4129.

- Ogata, K., 2009. Modern Control Engineering, Pearson. Prentice-Hall, Boston.

- Rojas-Cubides, Harvey et al., 2019. Sliding mode control assisted by GPI observers for tracking tasks of a nonlinear multivariable Twin-Rotor aerodynamical system, Control Engineering Practice. Elsevier Ltd, 88(172), pp. 1-15. 